\title{
SH3-Binding Motif
}

National Cancer Institute

\section{Source}

National Cancer Institute. SH3-Binding Motif. NCI Thesaurus. Code C14109.

Typically found in proteins involved in signaling pathways, the SH3-Binding Motif is a peptide sequence that mediates regulated high-affinity physical interaction (binding) with the SH3 (SRC Homology 3) domain in targ et protein partners. The SH3-binding motif often share proline-rich stretches containing a common PXXP motif. $(\mathrm{NCl})$ 